\title{
A Theoretical Game Approach for Two- Echelon Stochastic Inventory System
}

\author{
Saeed Alaei ${ }^{1}$, Alireza Hajji ${ }^{2}$, Reza Alaei ${ }^{2}$, Masoud Behravesh ${ }^{3 *}$ \\ ${ }^{1}$ Department of Industrial Engineering, K. N. Toosi University of Technology, \\ Pardis St., Molla-Sadra Ave., Vanak Sq., P.O. Box: 19991-43344, Tehran, Iran. \\ s.alaei@kntu.ac.ir, r.alaei@kntu.ac.ir \\ ${ }^{2}$ Department of Industrial Engineering, Sharif University of Technology, Azadi \\ Ave, P.O. Box: 11365-8639, Tehran, Iran, ahaji@sharif.edu \\ ${ }^{3}$ Young Researchers and Elite Club, Marand Branch, Islamic Azad University, \\ Kasrayi St., Daneshgah Sq., P.O. Box: 461/15655, Marand, Iran, \\ behravesh@marandiau.ac.ir (corresponding Author)
}

\begin{abstract}
In this paper, we study the differences between the Centralized and Decentralized approaches in a two-echelon stochastic inventory system under the lost sale policy. We formulate the condition in which the retailer applies $(r, Q)$ inventory policy, and his relation with the upper echelon who acts as a manufacturer. This situation has not been considered in the literature before. The Centralized approach results in optimal solution of the system and the Decentralized one is based on Stackelberg game in which the manufacturer is the leader. The demand arrives according to the stationary Poisson process. We drive the long-run average cost functions, then a set of computational steps are developed to obtain the solutions. Furthermore, we provide a numerical study to compare the two approaches. Here are some conclusions: (a) the Decentralized approach reduces the system's cost efficiency; (b) moreover, the Decentralized approach raises the lead time, the order quantity, and the supply chain inventory relative to the Centralized approach.
\end{abstract}

Keywords: Continuous Review Policy; Two-Echelon Inventory System; Inventory Management; Stackelberg Game

\section{Introduction}

Essentially, a supply chain is composed of independent members, each with its own objectives and individual costs. It is important how the members behave, to manage their inventory. If overall system performance is the objective, then choosing policies to minimize total costs, i.e., the optimal solution. Although this approach is appealing it has an important flaw. Each member may incur only a 
fraction of the supply chain cost. So, each member's costs may not be minimized in the optimal solution. For example, a supplier may care more than a retailer about consumer backorders for the supplier's product, or the retailer's cost to hold inventory may be higher than the supplier's [1].

While the firms may agree to cooperate in order to reduce overall system costs, each may face the temptation to deviate from any agreements, as to minimize their own costs. So, when each firm is interested in minimizing their own costs independently, it chooses policies, in which, the overall system performance, cannot necessarily be optimized. In this paper, we study the differences between the Centralized and Decentralized approaches in a two-stage serial supply chain including a manufacturer and a retailer. The Decentralized approach is based on a game theory model called the Stackelberg game.

There are two types of games considered in current literature: cooperative and non-cooperative games. In cooperative games, none of the players dominate the others, and the firms simultaneously choose their policies, While, in noncooperative games, this is not true.

Stackelberg game is a non-cooperative game. The research conducted in this paper presents a model of (1) the Centralized model in which, the goal is minimizing the overall system costs results in the optimal solution, and (2) the Decentralized or Stackelberg model, where individual firms in the supply chain have their own objectives and decisions to optimize. In this competitive approach, two firms play a game to achieve Stackelberg equilibrium. Stackelberg equilibrium is a pair of policies in which each firm minimizes its own cost assuming the other player chooses its equilibrium policy. Thus, each firm makes an optimal decision given the behaviour of the other firm, and therefore, none has an incentive to deviate unilaterally from the equilibrium.

We consider a supply chain with one manufacturer and a single retailer in our inventory system. An outside supplier supplies raw material to the manufacturer with zero lead time and the manufacturer produces a product at the rate of $\mu$ and supplies it to a retailer who in turn supplies it to the consumers. Furthermore, assume that the demands arrive according to a stationary Poisson process with the rate of $\lambda$ to the retailer. The retailer uses the continuous review $(r, Q)$ inventory policy for controlling costs. The manufacturer operates on a make-to-order basis and uses a lot-for-lot policy, and then he begins to produce a batch of $Q$, as soon as he receives an order from the retailer and delivers it to him after the lead time (l). Moreover, the manufacturer holds a monopolistic status and has an opportunity to obtain some inventory and demand information of the retailer, so he can gain advantage of this information for decreasing his own costs.

Hsiao et al. (2005) investigate the situation in which, the supplier and retailer choose the lead time and the cycle time respectively. They use Stackelberg game to analyse the problem [2]. 
In the Decentralized/Stackelberg approach, the manufacturer, as a leader, who is aware of the reaction of the retailer, optimizes his lead time, and the production rate. And as a follower, the retailer takes the manufacturer's optimal decisions as input parameters to determine order quantity and reorder points. This paper is organized as follows. Section 2 reviews the related literature with studies focused on non-cooperative and Stackelberg games. Section 3 describes the notations, and the long-run average cost structure describing the inventory patterns of the manufacturer and the retailer. Then we develop the Centralized and Stackelberg policies for the problem. Section 4 presents a set of computational algorithms in order to search Stackelberg equilibrium and the Centralized solution. Section 5 presents a numerical study and the corresponding sensitivity analysis for some parameters with the purpose of evaluating the influence of these parameters on costs, the manufacturer and the retailer decisions, and the competition penalty. Section 6 summarizes the results and provides concluding remarks.

\section{Literature Review}

In recent years, many papers have been published in the field of multi-echelon inventory management in which the game theory approach is adopted. We focus on studies in which non-cooperative and Stackelberg games have been investigated. There are some papers in the literature that study a vendor-buyer or seller-buyer supply chain, and they analyse the problem as a Stackelberg game in which the vendor/seller is the leader, and the buyer is the follower. Monahan (1984) and Chiang et al. (1994) consider the quantity discount model, in which, the vendor is willing to optimize his discount schedule [3, 4]. Eliashberg and Steinberg (1987) consider production activities such as product delivery and inventory policy, and their relation to marketing strategies such as pricing policies [5]. Hsiao and Lin (2005) discuss an EOQ model and investigate the optimal lead time and cycle time of the supplier and the retailer [2]. Qin et al. (2007) study a system in which demand is price-sensitive [6].

They compare the discounts that the supplier gives to the buyer in the centralized approach with the decentralized one. Liou et al. (2006) study multi-period inventory models in which the economic order quantity is integrated with the economic production quantity (EOQ-EPQ). They investigate the problem under the Stackelberg game approach and obtain the optimality conditions and the optimal replenishment policy [7]. Lal and Staelin (1984) investigate why and how a vendor should develop a pricing scheme, even if such a pricing structure does not change the consumer demand. They show that for any given pricing scheme, there exists, a unified pricing policy, which motivates the buyer to increase their order quantity per order [8]. Moreover, they show that the seller can reduce their costs while leaving the buyer in a stable condition. There are some papers in the literature that handle the problem studied by Lal and Staelin (1984) with the 
Stackelberg game approach [8]. For example, see (Rosenblatt and Lee, 1985; Weng, 1995; Munson and Rosenblatt, 2001; Wang, 2002) [9, 10, 11, 12]. Parlar and Wang (1994) investigate discounting decisions for a supplier with a group of homogeneous buyers. They use Stackelberg game and show that the seller set up his quantity discount schedule so that the buyer will order more than his Economic Order Quantity (EOQ) [13]. Moreover, both the seller and the buyer can gain considerably from quantity discount. Wang and Wu (2000) expand the work of Parlar and Wang (1994) to multiple heterogeneous buyers. They propose a discount pricing policy based on the percentage increase from a buyers' order quantity before discount $[14,13]$.

Li et al. (1995) investigate the advantages of cooperation in a seller-buyer inventory control system [15]. First, the relationship between the seller and the buyer modelled as a non-cooperative game. Then, they develop interactive game theory to address the system cooperation problem. Furthermore, the optimal system order quantity-pricing strategies are determined. Li et al. (1996) focus on advantages of improving buyer-seller cooperation in an inventory system [16]. Both cooperative and non-cooperative games are considered. They show that the order quantity and the total system profit are higher at cooperation than at noncooperation. Furthermore, the wholesale price is higher at non-cooperation than at cooperation. Cachon and Zipkin (1999) investigate a two-stage serial supply chain in which the two sides use Base Stock policy for managing their inventory [1]. They consider two games: In one game, the firms are committed to tracking echelon inventory and in the other game, they track local inventory. They also, discuss two Stackelberg games that the supplier is the leader in one game and is the follower in another game. They show that competition reduces efficiency, but raises the supply chain inventory. Viswanathan and Piplani (2001) study a onevendor, multi-buyer supply chain in which the vendor specifies common replenishment periods and offers a price discount to entice the buyers to replenish only at those time periods [17]. They investigate the problem under the Stackelberg game concept. So, the optimal replenishment period and the price discount are determined. Axsater (2001) studies a one-warehouse, multi-retailer supply chain in which the warehouse, as well as its local costs, pays a penalty cost for a delay at the warehouse to the retailer facing the delay. He uses Stackelberg game and shows that if the game is played constantly, the system will approach game equilibrium, but not necessarily, the systems optimal solution [18]. Bylka (2003) considers a vendor-buyer system and defines some conditional games. He shows that competitive approach does not necessarily reduce system efficiency. Some authors investigate a one-manufacturer, multi-retailer in Vendor Managed Inventory system by using a Stackelberg game [19]. Yu et al. (2009.a), discuss how the vendor can benefit in the system for increasing his own profit. They investigate that the Stackelberg equilibrium can be improved by using cooperative contracts [20]. Yu et al. (2009.b) investigate how a manufacturer and his retailers interact with each other with the purpose of optimizing their individual net profits. They consider advertising, pricing, and inventory decisions in their model. 
Furthermore, there are some other papers that applied Stackelberg game to analyse multi-echelon inventory systems [21]. For example, see (Gal-or, 1985; Dowrick, 1986; Lau and Lau, 2004; 2005; Yang and Zhou, 2006; Chu et al. 2006) $[22,23,24,25,26,27]$. Summarizing the brief review above, the supplier in a two-echelon supply chain, is often considered as a seller/vendor and there are only a few papers in which the supplier acts as a manufacturer. Moreover, in later systems, in the literature, the demand sometimes varies with price or is deterministic. In this sense, we formulate the condition in which the retailer applies $(r, Q)$ inventory policy, and his relation with the upper echelon who acts as a manufacturer. This situation has not been considered in the literature before.

\section{Model Formulation}

\subsection{Notation}

In this paper, we use a notation for representing the parameters and the decision variables to model the inventory management problem in a two-echelon supply chain with the Centralized and Decentralized approaches. We denote the Poisson probability with $p(j ; \lambda)=(\lambda t)^{j} \cdot e^{-\lambda t} / j !$ and its tail probability with $P(r ; \lambda)=$ $\sum_{j=r}^{\infty} p(j ; \lambda)$ subject to $(j=0,1,2 \ldots)$.

\section{Parameters:}

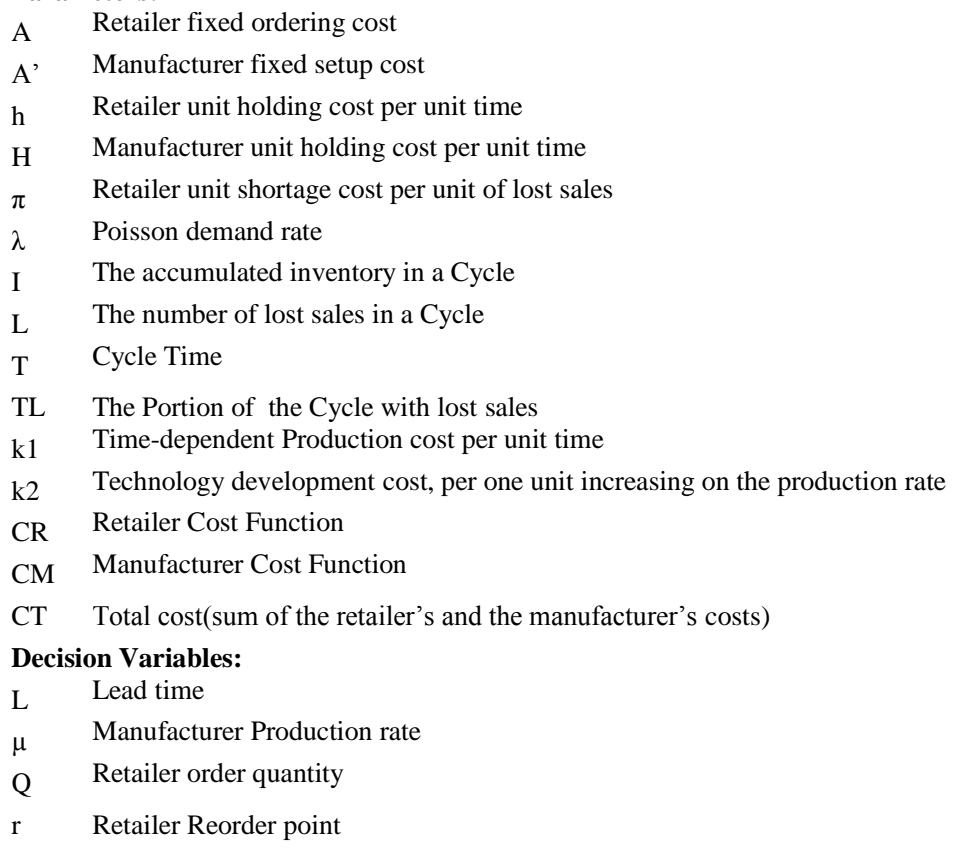




\subsection{Retailer Cost Model}

The retailer uses the continuous review (r, Q) inventory policy for controlling his costs in which $r$ and $Q$ are the reorder point and order quantity, respectively. We assume that the demand is according to stationary Poisson process, and unsatisfied demands are completely lost. The retailer costs include the holding cost, the fixed ordering cost, and the shortage cost. Figure 1 shows the retailer's inventory level. As shown in the figure, the cycle time $(\mathrm{T})$ is the time between two successive reorder times. We will assume that at most one order outstanding is allowed. In other words, when the retailer orders a batch of Q, he is not allowed to place another order, unless he received the previous deliverables. Under these conditions, the cycle time is always greater than or equal to the lead time.

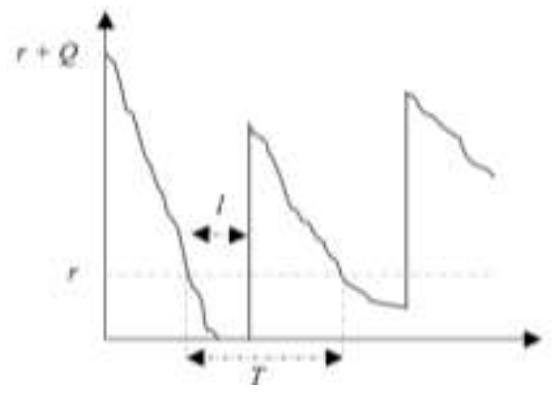

Figure 1

Retailer's Inventory Level

Rabinowitz et al. (1995), consider the partial backorder policy used in conjunction with the traditional (r, Q) inventory system [28]. Their policy is modelled using a control variable, $b$, which limits the maximum number of backorders allowed to be accumulated in any cycle. By setting $b$ to zero in their model, we have the lost sale policy. So, the expected cost for the retailer is given by:

$C_{R}=\frac{E(C)}{E(T)}=\frac{A+h \cdot E(I)+\pi \cdot E(L)}{E(T)}$

In which $\mathrm{T}$ is the cycle time, $\mathrm{C}$ is the costs accumulated within a cycle, $\mathrm{I}$ is the accumulated inventory in a cycle, and $\mathrm{L}$ is the number of lost sales in a cycle, and we have:

$$
\begin{aligned}
& E(L)=\lambda l P(r ; \lambda l)-r P(r+1 ; \lambda l) \\
& E(T)=\frac{Q}{\lambda}+l P(r ; \lambda l)-\frac{r}{\lambda} P(r+1 ; \lambda l) \\
& E(T)=\frac{Q}{\lambda}+l P(r ; \lambda l)-\frac{r}{\lambda}
\end{aligned}
$$

Where $i$ is given by:

$$
\begin{aligned}
& i=r l[P(r-1, \lambda l)-P(r, \lambda l)]+\lambda l^{2}[P(r-1, \lambda l)-P(r-2, \lambda l)] / 2+ \\
& r(r 1)[P(r+1, \lambda l)-P(r, \lambda l)] / 2 \lambda
\end{aligned}
$$


As long as $Q^{*} \geq r+1$, otherwise there is no feasible solution (Rabinowitz et al., 1995). According to the definition of Poisson's tail probability function and some of the properties of the Poisson distribution, it can be shown that $i$ is equal to zero.

\subsection{Manufacturer Cost Model}

The manufacturer Orders from an outside supplier with zero lead time. We consider a cost function for the manufacturer that consists of the holding cost, the setup costs, and time-dependent production cost. Holding cost is incurred only for finished products. We also split the setup costs into two parts: one part is fixed for every production period, and another one is an increasing function of the production rate. For example, assume an assembly line that has the technology for assembling a set of parts that are supplied by a supplier, and there is no cost for raw materials. In this assembly line, time-dependent production cost coincides with the daily production cost. If the production rate exceeds a specific limit, it is necessary to enhance the technology. For simplifying the problem, we assume that for every increasing unit on the production rate, the manufacturer incurred a cost called technology development cost. For instance, if the manufacturer incurred $200 \$$ for increasing 100 units on the production rate, then the technology development cost will be $2 \$$. Figure 2 shows the Manufacturer Inventory Level.

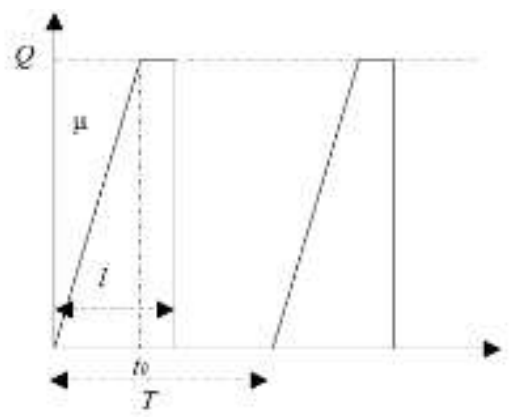

Figure 2

Manufacturer's Inventory Level

In this particular setting, the manufacturer's production cycle was equal to the retailer's replenishment Cycle. The manufacturer operates on a make-to-order basis using a lot-for-lot policy, So, begins to produce a batch of $\mathrm{Q}$ at the rate of $\mu$, as soon as he receives an order from the retailer and delivers it to him after the lead time. Let $T_{1}, T_{2}, \ldots$ be the sequence of reorder times of retailer, $C_{n}$ be the Reward at the time of the nth renewal, and $C(t)=\sum_{n=1}^{N(t)} C_{n}$ be the amount of cost incurred by $\mathrm{t}$, then, $\{C(t), t \geq 0\}$ forms a Renewal Reward Process where renewals occur at reorder times. Hence, the long-run average cost is given by:

$C_{M}=E(C) / E(T)=\left[A^{\prime}+H Q(2 l-Q / \mu) / 2+k_{1} Q / \mu+k_{2} \mu\right] / E(T)$ 
Remember that the amount of inventory held in a cycle is $Q(2 l-Q / \mu) / 2$; and $Q / \mu$ is the portion of the lead time that spent for production. The manufacturer makes a trade-off among inventory, setups, and production costs by producing at an optimal production rate. So, he spends a portion of lead time for production. Using a simple calculus, we can calculate the optimal amount of $\mu$ (given by the following equation) in order to minimize manufacturer cost.

$\mu^{*}=\left[Q\left(2 k_{1}-H Q\right) / 2 k_{2}\right]^{1 / 2}$

Consider that the production time ( $\mathrm{t} 0$ ) has to be lower than or equal to the lead time (See Figure 2). This results $\mu^{*}$ to be greater than or equal to $Q / l$. Furthermore, it is necessary that the right-hand side of the equation (7) to be a real number. Therefore, under these conditions, the equation (7) adjusted to the following equation:

$\mu^{*}=\left\{\begin{array}{c}{\left[\frac{Q\left(2 k_{1}-H Q\right)}{2 k_{2}}\right]^{\frac{1}{2}} ; \text { if } Q \leq \frac{2 k_{1} l^{2}}{2 k_{2}+H l^{2}}} \\ Q / l ; \text { otherwise }\end{array}\right.$

\subsection{Centralized Approach}

In the Centralized approach, the goal is minimizing the sum of manufacturer and retailer costs. Decision variables are reorder point (r), order quantity $(\mathrm{Q})$, lead time $(1)$, and production rate $(\mu)$. In this approach, we denote the Centralized solution with $\left(r^{*}, Q^{*}, l^{*}, \mu^{*}\right)$.

\subsection{Decentralized Approach (Stackelberg Game Approach)}

In a Stackelberg approach, players are classified as leader and follower. The leader chooses a strategy first, and then the follower observes this decision and makes his own strategy. It is necessary to assume that each enterprise is not willing to deviate from minimizing its own cost. In other words, each player chooses his best strategy. Here, the manufacturer is the leader, and the retailer is the follower. The manufacturer, as the Stackelberg leader, induces/encourages the retailer to choose his strategy by changing the value of lead time. So the manufacturer wants to find a set of $(\mathrm{r}, \mathrm{Q}, \mathrm{l})$ that minimizes his costs. In other words, the manufacturer determines his lead time, and acts as a leader by announcing lead time to the retailer in advance, and the retailer acts as a follower by choosing his reorder point and order quantity based on manufacturer strategy. We denote Stackelberg Equilibrium point with $\left(r^{s}, Q^{s}, l^{s}, \mu^{s}\right)$. The manufacturer and the retailer play a Stackelberg game to determine game Equilibrium. The two players cost functions are $C_{R}$ and $C_{M}$ (Equation 1 and 6). The manufacturer knows that if he sets his lead time value tol, then the retailer will set $\mathrm{r}$ and $\mathrm{Q}$ to values determined by his reaction function:

$[r(l), Q(l)]=\arg \min _{r, Q} C_{R}(r, Q, l)$ 
In other words, the retailer chooses $\mathrm{r}$ and $\mathrm{Q}$ that minimize his costs, given the manufacturer's strategy (1). Then the Manufacturer chooses $l^{*}$, its CostMinimizing lead time, given the reaction function of the retailer:

$$
\left[l^{s}, \mu^{s}\right]=\arg \min _{l, \mu} C_{M}(r(l), Q(l), l, \mu)
$$

\section{Search Algorithms}

In order to obtain the Centralized solution and Stackelberg equilibrium, we have four decision variables: $r, Q, l$, and $\mu$. As stated in previous sections, $\mu$ is an explicit function of $Q$ and $l$ (equation 9). In spite of the four-dimensional structure of the model, a three-dimensional search should be used to find the solutions. We assume that all variables but $\mu$ are integer numbers. Algorithms 1 and 2 summarize the computational steps for obtaining the centralized and Stackelberg solutions, respectively. Consider that the step 3 of both algorithms is associated to sub-algorithms 1 and 2, respectively.

Algorithm 1: Steps for obtaining Centralized solution

Step 1. Initialization: Set $l=0$

Step 2. Set $l=l+1$

Step 3. Calculate the optimal $r, Q$ and $\mu$ for current lead time $(l)$. And set them $r(l), Q(l)$ and $\mu(l)$ respectively (Sub-Algorithm 1)

Step 4. $\quad$ Calculate $C_{T}(Q(l), r(l), l, \mu(l))$ for current lead time $(l), r(l), Q(l)$ and $\mu(l)$

Step 5. If $l=1$, go to Step 2; otherwise go to the next step

Step 6. If $C_{T}(Q(l-1), r(l-1), l-1, \mu(l-1)) \geq C_{T}(Q(l), r(l), l, \mu(l))$, go to Step 2; otherwise stop. The Centralized solution will be

$$
\left(Q^{*}, r^{*}, l^{*}, \mu^{*}\right)=(Q(l-1), r(l-1), l-1, \mu(l-1))
$$

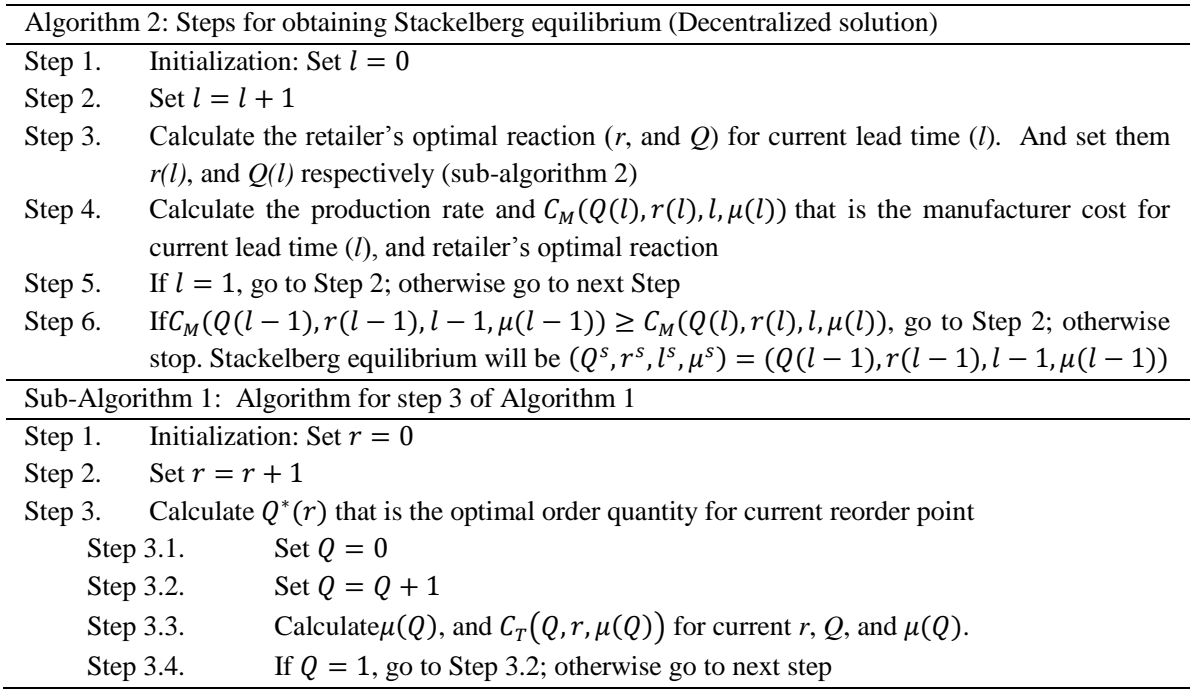


Step 3.5. If $C_{T}(Q-1, r, \mu(Q-1)) \geq C_{T}(Q, r, \mu(Q))$, go to Step 3.2; otherwise $Q^{*}(r)=$ $Q-1$, so, go to Step 4

Step 4. If $r=1$, go to Step 2; otherwise go to Step 5

Step 5. If $C_{T}\left(Q^{*}(r-1), r-1, \mu\left(Q^{*}(r-1)\right)\right) \geq C_{T}\left(Q^{*}(r), r, \mu\left(Q^{*}(r)\right)\right)$, go to Step 2; otherwise $r(l)=r-1$, and $Q(l)=Q^{*}(r-1)$, so, stop

Sub-Algorithm 2: Algorithm for step 3 of Algorithm 2

Step 1. Initialization: Set $r=0$

Step 2. Set $r=r+1$

Step 3. Calculate $Q^{*}(r)$ that is the optimal order quantity for current reorder point

Step 3.1. Set $Q=0$

Step 3.2. Set $Q=Q+1$

Step 3.3. Calculate $C_{R}(Q, r)$ that is the retailer's cost for current $r$ and $Q$.

Step 3.4. $\quad$ if $Q=1$, go to Step 3.2; otherwise go to next step

Step 3.5. If $C_{\mathrm{R}}(Q-1, r) \geq C_{R}(Q, r)$, go to Step 3.2; otherwise $Q^{*}(r)=Q-1$, so, go to Step 4

Step 4. if $r=1$, go to Step 2; otherwise go to Step 5

Step 5. $\quad$ if $C_{R}\left(Q^{*}(r-1), r-1\right) \geq C_{R}\left(Q^{*}(r), r\right)$, go to Step 2; otherwise $r(l)=r-1$, and $Q(l)=Q^{*}(r-1)$, so, stop.

\section{Numerical Study}

In this section, we present detailed numerical examples to:

- Compare the results of the Centralized approach with the results of Stackelberg game approach

- Evaluate the benefits of Stackelberg game approach to the manufacturer

- Illustrate Stackelberg game equilibrium graphically

- Analyse the sensitivity of the solutions, costs, and the competition penalty with respect to the variation of system parameters

\subsection{Base Case}

In our numerical study, we consider a base-case for implementing our algorithms. The parameter values for the base-case are presented in Table 1.

Table 1

Parameter values of the base-case

\begin{tabular}{|c|c|c|c|c|c|c|c|c|}
\hline Parameter & $A$ & $A^{\prime}$ & $h$ & $H$ & $\pi$ & $\lambda$ & $k 1$ & $k 2$ \\
\hline Value & 100 & 100 & 1 & 0.5 & 50 & 10 & 50 & 16 \\
\hline
\end{tabular}

Figure 3 shows the variation of manufacturer cost with respect to the lead time in the game approach. As shown in the figure, when the lead time is equal to 8 , the manufacturer cost is minimized. Furthermore, Figure 4 illustrates the variation of 
the total cost with respect to the lead time in the Centralized approach. When the lead time is equal to 2 , the total cost is minimized.

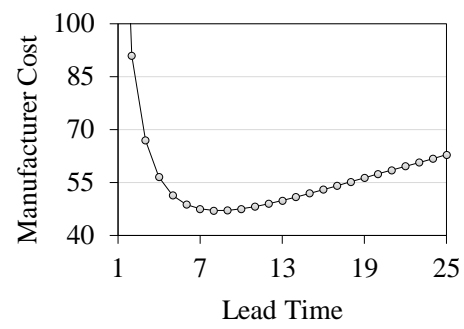

Figure 3

Manufacturer Cost in the Game Approach

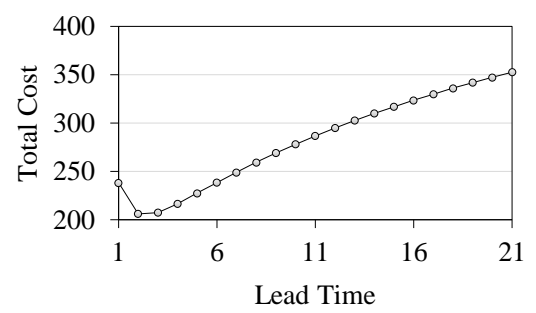

Figure 4

Centralized Total Cost

Table 2 summarizes the Centralized and Stackelberg solutions for the base-case values. From table 2, the lead time and order quantity have increased in the game approach, but the production rate has decreased, and the reorder point has not changed. The manufacturer cost is decreased by $48 \%$, and the retailer cost is increased by $84 \%$ in the game approach. So, the manufacturer gains the advantages of the game approach for decreasing his costs. However, the total cost in the game approach is increased by $26 \%$ subject to the centralized approach. This is known as the competition penalty.

Table 2

Solutions of two approaches for the Base-case Example

\begin{tabular}{|c|c|c|c|c|c|c|c|}
\hline & $l^{*}$ & $Q^{*}$ & $\mu^{*}$ & $r^{*}$ & $C R$ & $C M$ & $C T$ \\
\hline Centralized solution & 2 & 121 & 60.5 & 7 & 115.46 & 90.64 & 206.1 \\
\hline $\begin{array}{l}\text { Stackelberg } \\
\text { Equilibrium }\end{array}$ & 8 & 212 & 26.5 & 7 & 212.4 & 47.04 & 259.4 \\
\hline
\end{tabular}




\subsection{Stackelberg Equilibrium}

In this section, we illustrate the Stackelberg equilibrium point graphically for the base-case values. To simplify the problem, we will assume that the reorder point is constant and equals to 7 . Therefore, the manufacturer and the retailer play a Stackelberg game to determine $Q$ and $l$. As noted before, when the manufacturer sets his lead time value to $l$, then the retailer's reaction function will be as follows:

$Q(l)=\arg \min _{Q} C_{R}(Q, l)$

The manufacturer chooses $l^{*}$, given the reaction function of the retailer:

$l^{s}=\arg \min _{l} C_{M}(Q(l), l)$

In other words, the manufacturer wants to find a combination of the lead time and the order quantity that minimizes the costs in which the order quantity would be the reaction value of the retailer to the manufacturer strategy $(l)$. To analyse the problem graphically, remember that equilibrium will be where the retailer's reaction functions or $Q(l)$ is tangential to the manufacturer's ISO-cost curve. Consider that the ISO-cost curve consists of all combinations of $l$ and $Q$ that yield the same cost for the manufacturer. Figure 5 shows the Stackelberg equilibrium point for the base-case data. There are two ISO-cost curves in the figure. Remember that, the lower ISO-cost curve represents the higher levels of Cost. The Retailer's reaction function is tangential to the Manufacturer's ISO-cost curve with the value of 47.04 where $l$, and $Q$ are equal to 8 and 212, respectively. Then Stackelberg equilibrium point will be $\left(l^{s}, Q^{s}\right)=(8,212)$.

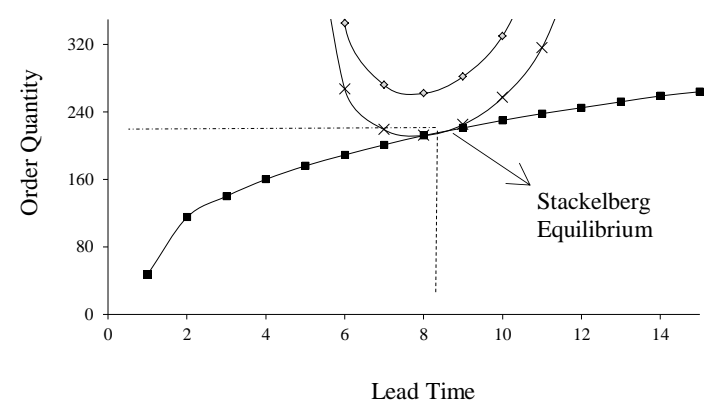

Figure 5

Stackelberg equilibrium and ISO-Cost Curves

\subsection{Sensitivity Analysis}

In this section, we perform a sensitivity analysis by changing the values of system parameters in the base-case. We solve a sample of 500 problems and then we draw the relevant conclusions in each sub section. 


\subsubsection{Variations of Optimal Solution and Game Equilibrium}

The Centralized solution and Stackelberg Equilibrium of the system along with the sensitivity analysis for all parameters are presented in Table 3 . We vary the parameters one at a time by doubling and halving the base-case values.

Table 3

Two approach solutions under variation of Base-Case Parameters

\begin{tabular}{|c|c|c|c|c|c|c|c|c|c|}
\hline \multirow{2}{*}{\multicolumn{2}{|c|}{$\begin{array}{l}\text { Solutions } \\
\rightarrow \\
\text { Parameters } \\
\downarrow\end{array}$}} & \multicolumn{4}{|c|}{ Centralized Solution } & \multicolumn{4}{|c|}{ Stackelberg Equilibrium } \\
\hline & & $l^{*}$ & $Q^{*}$ & $r^{*}$ & $\mu^{*}$ & $l^{s}$ & $Q^{s}$ & $r^{s}$ & $\mu^{s}$ \\
\hline & & 2 & 121 & 7 & 60.5 & 8 & 212 & 7 & 26.5 \\
\hline \multirow{3}{*}{$A$} & 50 & 2 & 117 & 7 & 58.5 & 8 & 210 & 7 & 26.25 \\
\hline & 20 & & & & & & & & \\
\hline & 0 & 3 & 152 & 7 & 50.7 & 8 & 215 & 7 & 26.9 \\
\hline \multirow{5}{*}{$A^{\prime}$} & 50 & 2 & 117 & 7 & 58.5 & 8 & 212 & 7 & 26.5 \\
\hline & 20 & & & & & & & & \\
\hline & 0 & 3 & 152 & 7 & 50.7 & 9 & 221 & 7 & 24.6 \\
\hline & 0.5 & 3 & 216 & 7 & 72 & 8 & 324 & 7 & 40.5 \\
\hline & 2 & 2 & 82 & 7 & 41 & 9 & 140 & 7 & 15.6 \\
\hline \multirow{3}{*}{$H$} & 0.2 & & & & & & & & \\
\hline & 5 & 2 & 121 & 7 & 60.5 & 11 & 238 & 7 & 21.6 \\
\hline & 1 & 2 & 120 & 7 & 60 & 6 & 189 & 7 & 31.5 \\
\hline \multirow{3}{*}{$\pi$} & 25 & 3 & 105 & 7 & 35 & 9 & 142 & 7 & 15.8 \\
\hline & 10 & & & & & & & & \\
\hline & 0 & 1 & 73 & 14 & 73 & 8 & 321 & 7 & 40.1 \\
\hline \multirow{2}{*}{$\lambda$} & 5 & 2 & 71 & 4 & 35.5 & 7 & 103 & 4 & 14.7 \\
\hline & 20 & 2 & 214 & 15 & 107 & 9 & 438 & 15 & 48.7 \\
\hline \multirow{3}{*}{$k 1$} & 25 & 2 & 117 & 7 & 58.5 & 9 & 221 & 7 & 24.6 \\
\hline & 10 & & & & & & & & \\
\hline & 0 & 2 & 128 & 7 & 64 & 7 & 201 & 7 & 28.7 \\
\hline \multirow{2}{*}{$k 2$} & 8 & 1 & 72 & 13 & 72 & 6 & 189 & 7 & 31.5 \\
\hline & 32 & 4 & 159 & 7 & 39.75 & 12 & 245 & 7 & 20.4 \\
\hline
\end{tabular}

Here are some representative observations:

(1) The decentralized approach raises the lead time and order quantity values, but lowers the values of the reorder point and production rate. In other words, we always have:

$$
l^{*}<l^{s}, \quad \mu^{*}>\mu^{s}, \quad Q^{*}<Q^{s}, \quad r^{*} \geq r^{s}
$$

This is because, in the Decentralized approach, the manufacturer wants to increase the cycle time in order to minimize related costs (see equation 6). They could only increase the lead time to achieve this goal. It can be proven that $d E(T) / d l=P(r, \lambda l) \geq 0$. As a consequence, in each cycle, he has more time for 
production, and then they could reduce the production rate. In the other hand, regarding to the increase on the cycle time, the retailer has to increase his order quantity. This reaction is in the same direction of the manufacturer goal, because $d E(T) / d Q=1 / \lambda \geq 0$. It is reasonable for the retailer to increase the reorder point regarding to increase on the lead time in order to reduce his shortage cost, but he acts vice versa. Because, increasing the reorder point is in opposition to the manufacturers' goal. It can be proved that the increasing one unit on the reorder point results in decreasing the cycle time by $P(r+1, \lambda l) / \lambda$. So if the retailer wants to increase the reorder point, the manufacturer increases the lead time to compensate the reduction on the cycle time.

(2) Furthermore, the decentralized approach raises the supply chain inventory relative to the centralized solution. In other words, if firms choose the optimal solution, they will tend to decrease inventory.

(3) The Manufacturer only spends a portion of lead time for production. This time is equal to $Q / \mu$. The remaining time $\left(l-t_{0}\right)$ is spent to holding inventory without production. The manufacturer delivers a batch as soon as possible after the production period in the Centralized approach. However, in the game approach, he prefers to deliver the batch very late in some cases. This result in increasing the retailer's cost.

\subsubsection{Variations of the Manufacturer and the Retailer Costs}

In the centralized approach, there is no inventory system if the lead time value is greater than 54. In other words, the total average cost of the retailer exceeds $\pi \lambda$, then it is reasonable that every order has been lost. In this approach, the maximum value of the order quantity is 320 . However, in the decentralized approach, for any value of the lead time, there exists an inventory system. The maximum value of the order quantity is 499 (also for infinite values of the lead time). This is because the holding cost for the $500^{\text {th }}$ unit and above values of the order quantity is greater than their shortage cost. Figure 6 shows the variations of the manufacturer and the retailer costs with respect to some selected parameters for two policies. $\mathrm{CM}$ and $\mathrm{CR}$ represent the manufacturer and the retailer cost, respectively. We select several representative observations summarized as follows:

(1) The manufacturer gains the advantages of the game approach for decreasing costs. However, the retailer cost in the game approach is always greater than its value in the centralized approach.

(2) In the centralized approach, the effects of increment in the shortage cost are tolerated by the manufacturer. However, in the game approach, the manufacturer chooses his strategy in a manner that his cost experiences no rise.

(3) The retailer cost increment is always greater than the manufacturer cost 
decrement in the game approach. For example, in the base-case, the game approach results in an increase of 97 units in the retailer cost, however, the manufacturer cost decrement is 43 units.
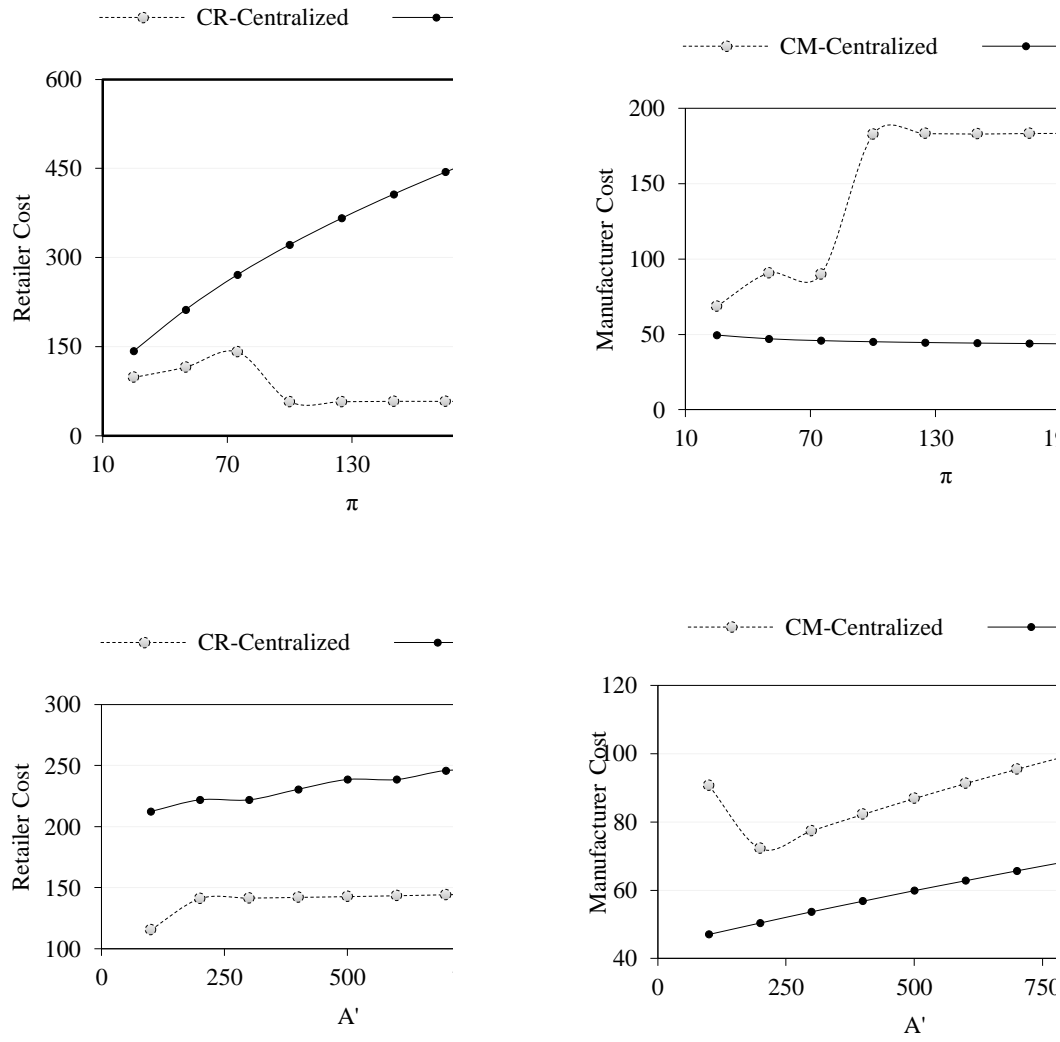

Figure 6

Variations of the manufacturer and the retailer costs in two approaches

\subsubsection{Variations of the Competition Penalty}

The competition penalty is the difference between the Centralized total cost and Stackelberg total cost measured as a fraction of the Centralized total cost. Figure 7 shows the variations of the competition penalty $(\rho)$ with respect to some selected parameters of the system. We select several representative observations summarized as follows:

(1) The value of $\rho$ is always positive. So, the total cost in the decentralized approach is always greater than that in the Centralized approach.

(2) The competition penalty is less sensitive to variations of $A^{\prime}$. 
(3) Shortage cost has the most significant influence on the competition penalty.
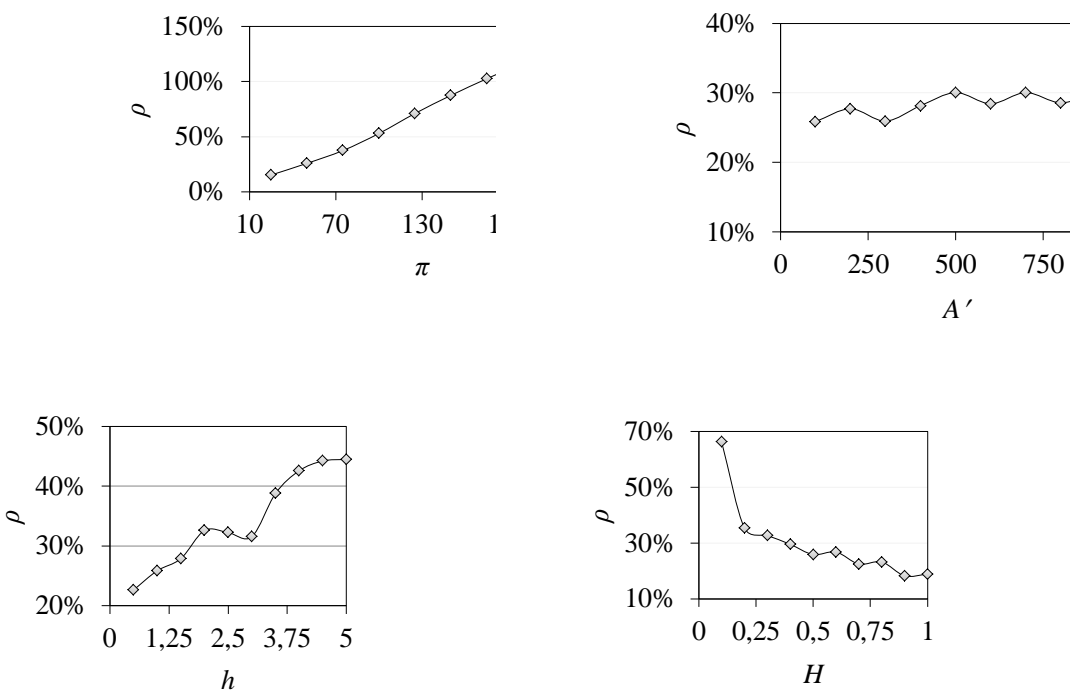

Figure 7

Variations of competition penalty with respect to various parameters

\section{Conclusions}

Whereas, the firms in a supply chain may agree to cooperate in order to minimize overall system costs, each firm may face the temptation to deviate from any agreement to reduce its own costs. This paper has studied the difference between the Centralized and decentralized approaches in a two-stage serial supply chain in which the manufacturer has the opportunity to obtain some inventory and marketrelated information of the retailer and then they can take advantage of this information for decreasing their own costs. We provided a numerical example and the corresponding sensitivity analysis for evaluating the costs, the competition penalty, and the manufacturer's and retailer's decisions in the two approaches. Here are some important results:

(1) The Decentralized approach reduces the systems cost efficiency.

(2) Shortage cost has the most significant influence on the competition penalty. For example, with the increase of the shortage cost from 50 in the base-case to 250 , the competition penalty increases from $26 \%$ to $150 \%$.

(3) The manufacturer gains the advantages of the game approach for decreasing their costs. However, the retailer cost increased in the game approach. Additionally, the retailer cost increment is always greater than 
the manufacturer cost decrement in the game approach. For example, in the base-case, the game approach results in an increase of 97 units in the retailer cost, but the manufacturer cost decrement is 43 units.

(4) In the Decentralized approach, manufacturer tends to increase the lead time and decrease the production rate. On the other hand, the retailer wants to increase the order quantity and doesn't want to increase the reorder point.

(5) The decentralized approach raises the supply chain inventory relative to the centralized approach. In other words, if firms choose the optimal solution, they will tend to decrease inventory. However, Cachon and Zipkin (1999) [1] suggest the opposite in a condition that two firms use base stock policy.

Our research could be extended in several possible directions. Because the decentralized approach reduces the system's cost efficiency, there is an opportunity for the players to coordination in order to align their incentives to reduce the supply chain's costs. Numerical studies indicate that the optimal solution is never a game equilibrium, so the firms can sign a contract with the purpose of achieving the system optimal solution, as in a game equilibrium. Moreover, we consider the lost sales policy in our inventory system, however, in some real inventory systems, it is more reasonable to assume that some of the excess demands are backordered, and the rest is lost. Then, we can extend the model in which a partial backorder policy is allowed.

\section{References}

[1] Cachon, G. P., Zipkin, P. H. (1999) Competitive and Cooperative Inventory Policies in a Two-Stage Supply Chain. Management Science, 45 (7): 936-953

[2] Hsiao, J. M., Lin, C. (2005) A Buyer-Vendor EOQ Model with Changeable Lead-Time in Supply Chain. International Journal of Advanced Manufacturing Technology, 26 (7-8): 917-921

[3] Monahan, J. P. (1984) A Quality Discount Pricing Model to Increase Vendor Profits. Management Science, 30 (6): 720-726

[4] Chiang, C. W., Fitzsimmons, J., Huang, Z., Li, Susan (1994) A Game Theoretic Approach to Quantity Discount Problem. Decision Sciences. 25 (1): $153-168$

[5] Eliashberg, J., Steinberg, R. (1987) Marketing-Production Decisions in an Industrial Channel of Distribution. Management Science, 33 (8): 981-1000

[6] Qin, Y., Tang, H., Guo, C. (2007) Channel Coordination and Volume Discounts with Price-Sensitive Demand. International Journal of Production Economics, 105 (1): 43-53

[7] Liou, Y. C., Schaible, S., Yao, J. C. (2006) Supply Chain Inventory Management via a Stackelberg Equilibrium. Journal of Industrial and 
Management Optimization, 2 (1): 81-94

[8] Lal, R., Staelin, R. (1984) An Approach for Developing an Optimal Discount Pricing Policy. Management Science, 30 (12): 1524-1539

[9] Rosenblatt, M. J., Lee, H. L. (1985) Improving Profitability with Quantity Discounts under Fixed Demand. IIE Transactions, 17 (4): 388-395

[10] Weng, Z. K. (1995) Channel Coordination and Quantity Discounts. Management Science, 41 (9): 1509-1522

[11] Munson, C. L., Rosenblatt, M. J. (2001) Coordinating a Three-Level Supply Chain with Quantity Discounts. IIE Transactions, 33 (5): 371-384

[12] Wang, Q. N. (2002) Determination of Suppliers' Optimal Quantity Discount Schedules with Heterogeneous Buyers. Naval Research Logistics, 49 (1): 46-59

[13] Parlar, M., Wang, Q. (1994) Discounting Decisions in a Supplier-Buyer Relationship with a Linear Buyer's Demand. IIE Transactions, 26 (2): 3441

[14] Wang, Q., Wu, Z. (2000) Improving a Supplier's Quantity Discount Gain from Many Different Buyers. IIE Transactions, 32 (11): 1071-1079

[15] Li, S., Huang, Z., Ashley, A. (1995) Seller Buyer System Cooperation in a Monopolistic Market. Journal of the Operational Research Society, 46 (12): $1456-1470$

[16] Li, S., Huang, Z., Ashley, A. (1996) Improving Buyer Seller System Cooperation through Inventory Control. International Journal of Production Economics, 43 (1): 37-46

[17] Viswanathan, S., Piplani, R. (2001) Coordinating Supply Chain Inventories through Common Replenishment Epochs. European Journal of Operational Research, 129 (2): 277-286

[18] Axsater, S. (2001) A Framework for Decentralized Multi-Echelon Inventory Control. IIE Transactions, 33 (2): 91-97

[19] Bylka, S. (2003) Competitive and Cooperative Policies for the VendorBuyer System. International Journal of Production Economics, 81-82 (11): 533-544

[20] Yu, Y., Chu F., Chen H. (2009a) A Stackelberg Game and its Improvement in VMI System with a Manufacturing Vendor. European Journal of Operational Research, 192 (3): 929-948

[21] Yu, Y., Huang G. Q., Liang L., (2009b) Stackelberg Game-Theoretic Model for Optimizing Advertising, Pricing and Inventory Policies in Vendor Managed Inventory (VMI) Production Supply Chains. Computers \& Industrial Engineering, 57 (1): 368-382 
[22] Gal-Or, E., (1985) First Mover and Second Mover Advantages. International Economic Review, 26 (3): 649-653

[23] Dowrick, S., (1986) Von Stackelberg and Cournot Duopoly: Choosing Roles. The Rand Journal of Economics, 17 (2): 251-260

[24] Lau, A. H. L., Lau, H. S. (2004) Some Two-Echelon Supply-Chain Games: Improving from Deterministic-Symmetric-Information to StochasticAsymmetric Information Models. European Journal of Operational Research, 161 (1): 203-223

[25] Lau, A. H. L., Lau, H. S. (2005) A Critical Comparison of the Various Plausible Inter-Echelon Gaming Processes in Supply Chain Models. The Journal of the Operational Research Society, 56 (11): 1273-1286

[26] Yang, S. L., Zhou, Y. W. (2006) Two-Echelon Supply Chain Models: Considering Duopolistic Retailers' Different Competitive behaviours. International Journal of Production Economics, 103: 104-116

[27] Chu, H., Wang, J., Jin, Y., Suo, H. (2006) Decentralized Inventory Control in a Two-Component Assembly System. International Journal of Production Economics, 102 (2): 255-264

[28] Rabinowitz, G., Mehrez, A., Chu, C. W., Patuwo, B. E. (1995) A Partial Backorder Control for Continuous Review of (r, Q) Inventory System with Poisson Demand and Constant Lead Time. Computers and Operations Research, 22 (7): 689-700 